Pacific Journal of Mathematics

CONTINUA IN THE PLANE WITH LIMIT DIRECTIONS

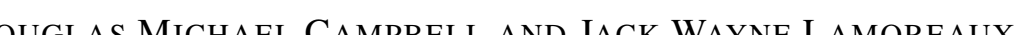




\title{
CONTINUA IN THE PLANE WITH LIMIT DIRECTIONS
}

\author{
Douglas Michael Campbell and Jack Lamoreaux
}

This paper is devoted to the analysis of sets in the plane which have at least one limit direction at each point. In the proof of a variational technique used in univalent function theory Schiffer used the fact that a continuum in the plane which only has limit directions \pm 1 is a horizontal segment, a result of Haslam-Jones which used measure theory and some rather unusual topological terminology. The attempt to find an elementary topological proof has led to several false proofs in the literature. Not only do we establish Haslam-Jones' result using only elementary topology but we obtain the conclusion under far weaker conditions allowing us to obtain a set theoretic counterpart to the real variable theorem that says if the upper right Dini derivative of a function is zero on an interval, then the function is constant on that interval. We then extend the result to allow for the possibility of exceptional points. Our strongest result gives a complete classification of continua with exceptional points. The paper closes with an open problem.

THEOREM. Let $E$ be a continuum in the plane and let $K$ be a subset of $E$ such that each point of $E-K$ has weak right limit direction 1. Then $E$ is a horizontal segment if and only if the projection of $K$ on the $y$-axis has Lebesque measure zero.

It is well known that if $f(x)$ is a continuous function on $[a, b]$ with $f^{\prime}(x)=0$ on $(a, b)$, then $f(x)$ is a constant, that is, the graph of $f(x)$ is a horizontal line segment. There are several strengthened versions of this fact. If $f(x)$ is absolutely continuous on $[a, b]$, then $f^{\prime}$ need only be zero almost everywhere in order that $f(x)$ be constant. If $f(x)$ is only continuous but $f^{\prime}(x)=0$ a.e., and $f^{\prime}(x)$ exists finitely at all but a countable number of points, then $f(x)$ is a constant. Finally, if the upper right Dini derivative of $f(x), D^{+} f(x)$, is equal to zero on $[a, b]$, then $f(x)$ is a constant [1].

We can ask if these results can be generalized to arbitrary plane sets. Let $E$ be a point set in the plane and $f$ be a mapping of $[0,1]$ onto $E$. Instead of saying that the derivative of the function $f:[0,1] \rightarrow E$ is zero at a point, it would be nice to talk about the set $E$ itself and consider the set $E$ being "flat" at a point. This leads to the definition of a limit direction of a set at a point.

Definition. Let $E$ be a set in the complex plane, $z$ be a point of 
$E$, and $\alpha$ be a complex number of modulus 1 . A set $E$ is said to have a limit direction $\alpha$ at $z$ if and only if there is a sequence of points $z_{n}$ in $E-\{z\}$ which converges to $z$ with $\lim _{n \rightarrow \infty}\left(z_{n}-z\right) /\left|z_{n}-z\right|=\alpha$.

This paper is devoted to the analysis of sets in the plane which

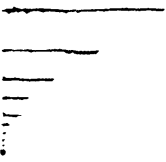

$$
\bigcup_{n=1}^{\infty}\left\{(x, y): y=\frac{1}{n}, 0 \leqq x \leqq \frac{1}{n}\right\} \cup\{0,0\}
$$

Figure 1

(a)

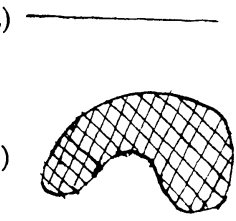

(c)

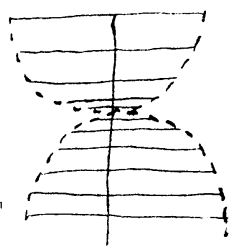

(d)

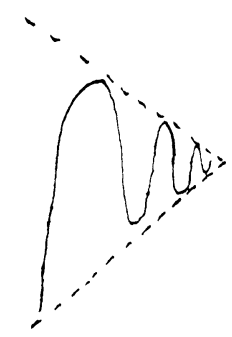

(e)

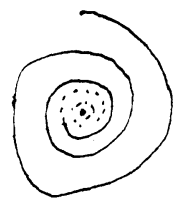

(f)

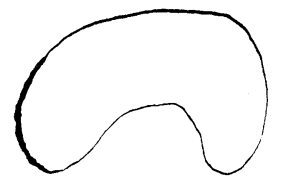

(g)

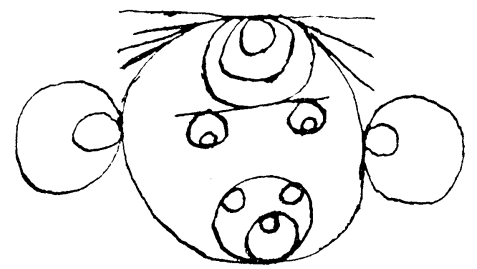

(h)

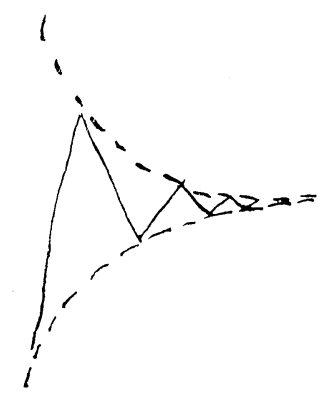

(i)

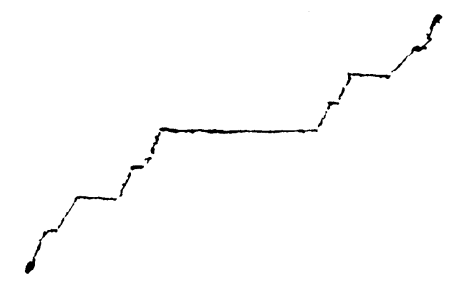


have at least one limit direction at each point. Let us see how this reduces to the study of nondegenerate continua.

If $E$ is a set in the plane which has a limit direction at each of its points, then each point of $E$ is itself a limit point of $E$ and $E$ can have no isolated points. Since we are primarily interested in the limiting directions of $E$ let us assume that $E$ is closed, hence perfect. By writing $E$ as the union of its components we have reduced the problem to the study of single points (degenerate continua) and closed connected sets containing more than one point (nondegenerate continua). Thus the classification of sets in the plane which have a limit direction at every point reduces to a classification of nondegenerate continua.

Figure 1 shows a perfect set which has a degenerate component at the origin. The examples of Figure 2 suggest the variety of possible behavior of limit directions of continua.

If a plane point set $E$ is the graph of some function $f(x)$, then the simple requirement $f^{\prime}(x)=0$ forces $E$ to be a horizontal line segment. We wish to find equally simple requirements in terms of the set $E$ itself which will force $E$ to be a horizontal line. Let us gain some clues by examining other parts of Figure 2.

Of course if the interior of a continuum $E$ is nonempty, then there are points of $E$ with every possible limit direction (Figure $2 b$ ). However, a continuum can have every possible limit direction at some $z$ in $E$ and still have empty interior (Figure 2c, Figure 2e). Demanding that each point have at most a countable number of limit directions does not help (Figure $2 \mathrm{~h}$ ) but it does eliminate some homeomorphic images of $[0,1]$ (Figure 2d, Figure 2e). One might hope the stringent restriction that at each point of $E$ there are at most two limit directions would force the set to be like Figure $2 \mathrm{f}$ or Figure 2h. This is not the case as forms of Figure $2 \mathrm{~g}$ show. Finally it does not suffice to demand that $E$ have at most limit directions \pm 1 for all points except a set of measure zero since the graph of the well known Cantor function (Figure 2i) is a nondegenerate continuum with at most limit directions \pm 1 for all points except a set with projection on the $x$-axis of measure zero. Nevertheless, with suitable restrictions we can establish the truth of some of our intuitive feelings.

THEOREM 1. If $E$ is a continuum in the plane which at each of its points has at most the two limit directions +1 and -1 then $E$ is a horizontal segment.

Schiffer used Theorem 1 in the proof of his variational lemma which is used in univalent function theory [5]. The proof depended 
on a result of Haslam-Jones [2] which used measure theory and some rather unusual topological terminology. In 1966 and then in 1969 Huckemann [3], [4], attempted an elementary proof but both his papers contained fundamental errors.

Before we give the proof of Theorem 1 let us first agree on some notation and state an obvious lemma. Let $D(a, b)$ denote a disc centered at $(a, b)$. Let $R\left(x_{1} x_{2}\right)$ be the closed rectangle $\left\{(x, y): x_{1} \leqq x \leqq x_{2}\right.$, $0 \leqq y \leqq 1\}$. By a sector of angular opening $\varepsilon$ centered at $(a, b)$ we mean $\{(a, b)\} \cup\{(x, y): \mid \arctan ((y-b) /(x-a)) \leqq \varepsilon\}$. We denote this sector by $S_{\varepsilon}(a, b)$.

Lemma 1. If $\left\{\left(x, y_{i}\right)\right\}, i=1, \cdots, m$ is a finite set of distinct points in $[0,1] \times(0,1)$ and $d=\min \left[1-y_{m}, \cdots, y_{2}-y_{1}, y_{1}-0\right]$, then for any $\varepsilon$ less than arctan $(d / 4)$ the sectors $S_{\varepsilon}\left(x, y_{i}\right)$ are separated inside $[0,1] \times[0,1]$ by a distance of at least $d / 2$.

Proof of Theorem 1. Without loss of generality we may suppose that $E$ is contained in the unit square $[0,1] \times[0,1]$, that $E$ has no points on the lines $y=0$ and $y=1$, and that $E$ has at least one point on each of the lines $x=0$ and $x=1$.

We now prove that $E$ must be a single horizontal line segment. Let $x_{1}=0$ and let $A_{1}=\left\{\left(x_{1}, y_{i}^{1}\right)\right\}$ be the finite nonempty set of points of $E$ on the line $x=x_{1}$ (if $A_{1}$ were infinite, then $E$ would have $\pm i$ as a limit direction). Let $\varepsilon>0$ be given. Choose $0<\varepsilon_{1}<\varepsilon$ so that Lemma 1 is satisfied for the sectors $S_{\varepsilon_{1}}\left(x_{1}, y_{i}^{1}\right)$ and let $S_{1}=\bigcup S_{\varepsilon_{1}}\left(x_{1}, y_{i}^{1}\right)$. Since at each point of $E$ the only limit directions are \pm 1 , we can choose disks $D\left(x_{1}, y_{i}^{1}\right)$ small enough so that each $D\left(x_{1}, y_{i}^{1}\right) \cap E$ is a subset of $S_{1}$. Since $E-\bigcup_{i} D\left(x_{1}, y_{i}^{1}\right)$ is a compact set which does not intersect $x=x_{1}$, then there exists an $r>x_{1}$ such that $E \cap R\left(x_{1}, r\right) \subset S_{1}$.

If $x_{2}=\operatorname{LUB}\left\{r: E \cap R\left(x_{1}, r\right) \subset S_{1}\right\}$ were 1 for every $\varepsilon>0$, then from the connectivity of $E$ there would only be one point in the set $A_{1}$ and $E$ would be a single horizontal segment as claimed.

We therefore suppose that for some $\varepsilon>0, x_{1}<x_{2}<1$. We now proceed to define a sequence of points in $E$ inductively. We replace $x_{1}$ by $x_{i}$. We let $A_{i}$ be the finite nonempty set of points of $E$ on the line $x=x_{i}, S_{i}$ be the union of sectors on $A_{i}$ of size $\varepsilon_{i}$, and $x_{i+1}=\operatorname{LUB}\left\{r: E \cap R\left(x_{i}, r\right) \subset S_{i}\right\}$. If at any stage $x_{i}$ were equal to 1 , then $E$ would be disconnected contrary to hypothesis. Hence we create an infinite monotone increasing sequence $\left\{x_{n}\right\}$ converging to some point $x_{0} \leqq 1$.

We say that a point $p$ of $A_{i}$ is a beginning point if $p$ is not a limit point of $S_{i-1} \cap E$ (all points of $A_{1}$ are defined to be beginning points). All other points of $A_{i}$ are called descendent points. We say that $q$ is a descendent of $p$ if $p$ is in some $A_{i}, q$ is in some $A_{i+k}$ and 
there are points $p=p_{i}, p_{i+1}, \cdots, p_{i+k}=q$ such that $p_{j} \in A_{j}, j=i, \cdots$, $i+k$, and $p_{j}$ is a limit point of $E \cap S_{\varepsilon_{j-1}}\left(p_{j-1}\right), j=i+1, \cdots, i+k$. Our construction of the sets $A_{i}$ and the connectivity of $E$ guarantee that

(1) no point in $A=\bigcup_{i=1}^{\infty} A_{i}$ can be the descendent of two different beginning points,

(2) each $A_{i}$ contains at least one beginning point,

(3) if $p$ is a beginning point of $A_{i}$, then $p$ has a descendent in $A$ for each $j>i$.

Thus, each beginning point is associated with an infinite set of descendents in $E$ which accumulate only at the line $x=x_{0}$. Since $E$ is compact this accumulation point is in $E$. Consequently, we can associate with each beginning point a point of $E$ on the line $x=x_{0}$. Furthermore, since the descendents of any two beginning points are separated by a positive distance bounded away from zero, each beginning point is associated with a unique point of $E$ on the line $x=x_{0}$. This forces the existence of an infinite number of points of $E$ on the line $x=x_{0}$ and violates the hypothesis that $E$ has only \pm 1 as a limit direction. This concludes the proof of the theorem.

A careful examination of the proof of Theorem 1 reveals we never used the fact that there is a two sided control on the limit direction at each point of $E$. All that was needed were the facts that $E$ does not have an infinite number of points on any vertical line and that if $(x, y)$ is in $E$, then for any sector $S$ opening to the right from $(x, y)$ there is a disc $D(x, y)$ such that $D(x, y) \cap E \cap R[x, 1]$ is a subset of $S$. Thus we are led to the following definition.

Definition. Let $z_{0}$ belong to a point set $E$ in the plane. The set $E$ has a right limit direction $\alpha$ at $z_{0}$ if and only if there is a sequence of points $\left\{z_{n}\right\}$ in $\left(E-\left\{z_{0}\right\}\right) \cap\left\{z: \operatorname{Re} z \geqq \operatorname{Re} z_{0}\right\}$ which converges to $z_{0}$ and for which $\lim _{n \rightarrow \infty}\left(z_{n}-z_{0}\right) /\left|z_{n}-z_{0}\right|=\alpha$.

THEOREM 2. If $E$ is a plane continuum with the property that for each point $z$ in $E$ either there is no right limit direction or there is only right limit direction 1 , then $E$ is a horizontal line segment.

Proof. The proof is a simple modification of the proof of theorem 1. Note that the connectivity of $E$ guarantees that at any of the points of $E$ on the line $x=x_{n}$ there must be a right limit direction.

Theorem 2 can be viewed as the set theoretic counterpart to the real variables theorem which says that if $D^{+} f(x)=0$ on $(0,1)$, then $f(x)$ is a constant. 
We now turn to the problem of exceptional points. It may be that $E$ is defined by a limiting process and consequently there may be no information available about the limit directions for some subset $K$ of $E$. One immediately asks how large this set of exceptional points can be and still have Theorems 1 or 2 hold.

We remind the reader of an elementary result of point set topology.

LEMMA $2[7, \mathrm{p} .13]$. Let $N$ be a nondegenerate continuum in the plane. Let $G$ be the annulus $\{z: a<|z|<b\}$. If $G$ is such that there are points of $N$ both inside and outside of $G$, then any component of $G \cap N$ must intersect either $|\boldsymbol{z}|=a$ or $|\boldsymbol{z}|=b$.

THEOREM 3. Let $E$ be a continuum in the plane and $p$ be a point of $E$. If there is a deleted neighborhood of $p$ such that all points of $E$ in this neighborhood have limit direction either \pm 1 , then $p$ can only have limit directions \pm 1 . Furthermore, $p$ must have at least +1 or -1 as a limit direction.

Proof. Without loss of generality we may assume that $p$ is the origin and that $0<|z|<\varepsilon$ is the deleted neighborhood. If for every $\varepsilon_{1}$ in $\varepsilon / 2<\varepsilon_{1}<3 \varepsilon / 4$ the point $i \varepsilon_{1}$ or the point $-i \varepsilon_{1}$ were an element of $E$, then, contrary to hypothesis, the neighborhood $0<|z|<\varepsilon$ would contain a point of $E$ with limit direction $\pm i$. We can therefore find a number $\varepsilon_{1}, 0<\varepsilon_{1}<\varepsilon$, such that the intersection of $E$ with the circle $|z|=\varepsilon_{1}$ does not contain either $\pm i \varepsilon_{1}$.

We claim that the intersection of $E$ with $|z|=\varepsilon_{1}$ is finite. Otherwise $E$ would contain an accumulation point $q \neq \pm \varepsilon_{1} i$ on $|z|=\varepsilon_{1}$. But this would mean that $q$ would belong to $E$, be within $0<|z|<\varepsilon$ and have limit direction other than \pm 1 , contrary to hypothesis.

We prove that the interection of $E$ and $|z|<\varepsilon_{1}$ consists of a finite number of horizontal line segments. First pick any point $z_{0}$ of $E$ which is not on the $x$-axis but is in the disc $|z|<\varepsilon_{1}$. Consider the annulus

$$
A=\left\{z:\left|\operatorname{Im} z_{0}\right| / 2<|z|<\varepsilon_{1}\right\} .
$$

Let $\mathfrak{A}$ denote the closure of the component of $A \cap E$ containing $z_{0}$. Clearly, $\mathfrak{A}$ is a nontrivial compact connected set and each point of $\mathfrak{A}$ has limit direction at most \pm 1 . Therefore, by Theorem $1 \mathfrak{A}$ is a horizontal line segment containing $z_{0}$. But by Lemma 2, $\mathfrak{A}$ must intersect either the circle $|z|=\left|\operatorname{Im} z_{0}\right| / 2$ or the circle $|z|=\varepsilon_{1}$. Since the segment does contain $z_{0}$, whose imaginary part is nonzero, the line segment can not intersect the circle $|z|=\left|\operatorname{Im} z_{0}\right| / 2$ and must 
therefore intersect the circle $|z|=\varepsilon_{1}$. But we have already shown that there are only a finite number of points of $E$ on $|z|=\varepsilon_{1}$. This proves that there are only a finite number of components of $E \cap$ $\left\{z:|z|<\varepsilon_{1}\right\}$ and that each is contained in a horizontal line segment. Therefore, the only possible limit directions at the origin are \pm 1 . Since $E$ is connected and the origin belongs to $E$ there must be at least one limit direction at the origin. This concludes the theorem.

In order to motivate the next definition consider the following. Let $E$ be a continuum in the plane and $K$ a subset of $E$. If $K$ is finite and each point of $E-K$ has only \pm 1 as limit directions, then a finite number of applications of Theorem 3 shows that $E$ is still a line segment. Furthermore, if $K$ is infinite but only has a finite number of accumulation points, then we can use Theorem 3 to remove the nonaccumulation points leaving only a finite set of exceptional points which, as we have already seen, is then removable. In order to extend this procedure let us recall the notion of the $n$th derived set.

Definition. Let $X$ be a set of points. Define $X^{(0)}$ to be $X$. If $n$ is a positive integer, then $X^{(n)}$, the $n$th derived set of $S$, is the set of accumulation points of $X^{(n-1)}$.

THEOREM 4. Let $E$ be a continuum in the plane and $K$ be a subset of $E$. Suppose that for every point of $E-K$ the only limit directions are \pm 1 . If for each point $p$ in $K$ there is a neighborhood $N$ of $p$ and a natural number $\underline{n}$ such that the $n$th derived set of $K \cap N$ is empty, then $E$ is a horizontal line segment.

Proof. Because of Theorem 1, $E$ will be a horizontal line segment if we can prove that any point of $K$ can only have \pm 1 as a limit direction. We proceed by induction on $\underline{n}$ and show that if $p$ is in $K$ and $N$ is a neighborhood of $p$ such that the $n$th derived set of $N \cap K$ is empty, then $p$ can only have \pm 1 as limit directions.

The case $n=1$ follows from Theorem 3. Assume that the statement is true for $n$. Let $p$ be a point of $K$ and $N$ a neighborhood of $p$ such that the $(n+1)$ derived set of $N \cap K$ is empty. If $p$ is

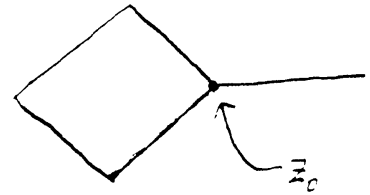

(a)

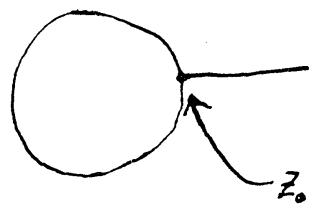

(b)

Figure 3 
in $K-K^{(1)}$, then by restricting the neighborhood $N$ and applying Theorem 3 we see that $p$ can only have limit direction \pm 1 . On the other hand, if $p$ is in $K^{(1)}$, then $K^{(1)}$ is a subset of $E$ and the $n$th derived set of $N \cap K^{(1)}$ is empty. Therefore, by the induction hypothesis, $p$ can only have the limit directions \pm 1 .

We now introduce our last definition preparatory to the concluding theorems.

Definition. A set $E$ in the plane is said to have a weak right limit direction 1 at $z_{0}$ an element of $E$ if and only if $E$ has no limit direction $e^{i \theta}, \theta \in[-\pi / 2,0) \cup(0, \pi / 2]$, at $z_{0}$.

Obviously if $E$ has limit directions at most \pm 1 at a point $z_{0}$ of $E$, then $E$ has weak right limit direction 1 at $z_{0}$. The converse in trivially false as Figure 3a shows. Furthermore, a set can have a right limit direction 1 at $z_{0}$ and yet not have weak right limit direction 1 at $z_{0}$ (Figure $3 \mathrm{~b}$ ).

THeOREM 5. Let $E$ be a continuum in the plane and let $K$ be a countable subset of $E$. If every point of $E-K$ has a weak right limit direction 1, then $E$ is a horizontal line segment.

Proof. We modify the proof of a theorem due to J. A. Hummel [6, p. 188]. The projection of a set $A$ on the $y$-axis is denoted by $\pi A$ and one dimensional Lebesque measure is denoted by $m$.

Since $E$ is a continuum its projection $\pi E$ is an interval. If suffices to prove $m(\pi E)=0$. Preceeding by contradiction we may suppose $m(\pi E)>0$.

We first define for any point $z_{0}$ the butterfly wing set

$$
S_{n}\left(z_{0}\right)=\left\{z: 0<\left|z-z_{0}\right|<\frac{1}{n}, \frac{\pi}{4}<\left|\arg \left(z-z_{0}\right)\right|<\frac{\pi}{2}+\frac{1}{n}\right\} .
$$

Since each point of $E-K$ has weak right limit direction 1 we see that we can write $E$ as $K \cup \bigcup_{n=1}^{\infty} B_{n}$ where $B_{n}=\left\{z \in E: S_{n}(z) \cap E=\varnothing\right\}$.

To show each $B_{n}$ is compact it suffices to show each $B_{n}$ is closed. If some $B_{n}$ were not closed, then it would be possible to find a sequence $z_{k}$ in $B_{n}, z_{k} \rightarrow z_{0}, z_{0} \in B_{n}$. This would imply the existence of a point $\zeta$ in $S_{n}\left(z_{0}\right) \cap E$. Since $S_{n}\left(z_{0}\right)$ is open there would exist a $\rho>0$ such that $\{z:|z-\zeta|<\rho\}$ would be contained in $S_{n}\left(z_{0}\right)$. But for any $z_{k}$ satisfying $\left|z_{k}-z_{0}\right|<\rho$ we would have $\zeta$ in $S_{n}\left(z_{k}\right) \cap E$ which contradicts our choice of $z_{k}$.

Because $K$ is countable $m\left(\pi \bigcup_{n=1}^{\infty} B_{n}\right)=m(\pi E)>0$. Therefore there is a number $N$ such that $m\left(\pi B_{N}\right)>0$. We turn to the problem of creating a geometrically manegeable subset of $B_{N}$. Let $D(z ; \rho)$ 
denote the open disc centered at $z$ with radius $\rho$. Since $B_{N}$ is compact and the collection $\left\{D(z, 1 / 2 N): z \in B_{N}\right\}$ covers $B_{N}$, there exists a finite subcollection which covers $B_{N}$ and hence a point $z_{0}$ in $B_{N}$ such that $m\left[\pi\left(D\left(z_{0} ; 1 / 2 N\right) \cap\left(B_{N}-K\right)\right)\right]>0$. To simplify notation let $L=$ $D\left(z_{0} ; 1 / 2 N\right) \cap\left(B_{N}-K\right)$.

If $z$ is in $B_{N}$, then from the definition of $B_{N}$ there are no points of $E$ within a distance of $1 / N$ which have the same $x$ coordinate as $z$. Thus if $z_{1}$ and $z_{2}$ are elements of $L$, then $\left|z_{1}-z_{2}\right|<1 / N$ and each is an element of $B_{N}$. Therefore they must have different $x$ coordinates. That is, $L$ is a graph over its projection on the $x$-axis.

In order to use the fact that all points of $E-K$ have a weak right limit direction 1 we choose $\varepsilon$ so that

$$
\frac{2}{N} \tan \varepsilon=\frac{1}{4} m[\pi(L)] \text {. }
$$

We now create a collection of sets whose projections form a Vitali covering of $\pi(L)$. Since each element $z^{\prime}$ of $L$ is an element of $E-K$, there exists a number $M$ such that if $z$ is in $D\left(z^{\prime} ; 1 / M\right) \cap E$ then $z$ is not in the sector from $\varepsilon$ to $\pi / 2$ nor the sector from $-\pi / 2$ to $-\varepsilon$. Let $\mathfrak{B}$ denote the collection of all sets of the form

$$
R_{\tilde{\delta}}\left(z_{1}\right)=\left\{z:\left|\arg \left(z_{1}-z\right)\right| \leqq \varepsilon \text { and } 0 \leqq x-x_{1} \leqq \delta\right\}
$$

where $z_{1}=x_{1}+i y_{1}$ runs through the elements of $L$ and $\delta$ runs through the numbers $0<\delta<1 / 2 M$ ( $M$ chosen as above for $z^{\prime}$ ). Clearly $\pi \mathfrak{B}$ is a Vitali cover of $\pi L$ and by the Vitali covering theorem there is a finite number of the sets $R_{\delta_{j}}\left(z_{j}\right)=R_{j}, j=1, \cdots, n$ such that the sets $\pi R_{j}$ are disjoint and

$$
\sum_{j=1}^{n} m\left[\pi\left(R_{j}\right)\right]>\frac{1}{2} m[\pi(L)] .
$$

Since each point of $L$ has a different $z$ coordinate and the sets $\pi\left(R_{j}\right)$ are disjoint we may index the points $z_{j}$ so that their $x$ coordinates are ordered $x_{1}<x_{2}<\cdots<x_{n}$. We now prove that $\delta_{i} \leqq x_{i+1}-x_{i}$. The diameter of $L$ is $1 / N$ and therefore $\left|z_{i}-z_{i+1}\right|<1 / N$. But if $z_{i}$ is in $B_{N}$ and $\left|z_{i}-z_{i+1}\right|<1 / N$, then the definition of $B_{N}$ forces $\left|y_{i+1}-y_{i}\right|<x_{i+1}-x_{1}$ which implies $\left|z_{i}-z_{i+1}\right|<\sqrt{2}\left(x_{i+1}-x_{i}\right)$. If we were to assume by way of contradiction that $x_{i+1}-x_{i}<\delta_{i}$, then $\left|z_{i}-z_{i+1}\right|<\sqrt{2}\left(x_{i+1}-x_{i}\right)$ would imply $z_{i+1}$ is in $N\left(z_{i}, 2 \delta_{i}\right)$ which is contained in $N\left(z_{i}, 1 / M_{i}\right)$ since $0<\delta_{i}<1 / M_{i}$ by construction. By the choice of $M_{i}$ we must therefore have $\left|\arg \left(z_{i}-z_{i+1}\right)\right|<\varepsilon$. But $\left|\arg \left(z_{i}-z_{i+1}\right)\right|<\varepsilon$ and $x_{i+1}-x_{i}<\delta_{i}$ would imply $\pi R_{i}$ and $\pi R_{i+1}$ are not disjoint which is contrary to construction.

Since $\delta_{i} \leqq x_{i+1}-x_{i}$ we have $\sum_{i=1}^{n} \delta_{i} \leqq$ diameter of $L<1 / N$. We 
now obtain a contradiction to the assumption that $m[\pi(L)]>0$. Using $(1 / 2) m[\pi(L)]<\sum_{j=1}^{n} m\left[\pi\left(R_{j}\right)\right]$ from the Vitali covering, the fact that $m\left[\pi\left(R_{j}\right)\right]=2 \delta_{j} \tan \varepsilon$ by construction, and the fact that $\sum_{j=1}^{n} \delta_{j}<1 / N$, we see that

$$
\frac{1}{2} m[\pi(L)]<\sum_{j=1}^{n}(2 \tan \varepsilon) \delta_{j}<\frac{2}{N} \tan \varepsilon<\frac{1}{4} m[\pi(L)] .
$$

This is impossible. This concludes the proof of the theorem.

A careful examination of the proof shows the following theorem on exceptional sets is true.

THEOREM 6. Let $E$ be a continuum in the plane and let $K$ be a subset of $E$. Suppose that every point of $E-K$ has weak right limit direction 1. Then $E$ is a horizontal line segment if and only if the projection of $K$ on the $y$-axis has measure zero.

We leave as an open problem the question whether Theorem 6 holds if for every point in $E-K$ the only right limit direction is 1 . We also ask if there is a proof for Theorem 5 which uses topological rather than measure theoretic tools.

\section{REFERENCES}

1. R. Boas, A Primer of Real Functions, Carus Monograph Series Volume 13, Math. Assoc. Amer. (1960).

2. U. S. Haslam-Jones, Tangential properties of a plane set of points, Quart. J. Math., 7 (1936), 116-123.

3. F. Huckemann, On Schiffer's variational lemma, Arch. Rational Mech. Anal., 22 (1966), 310-312.

4. — On Schiffer's variational Lemma II, Arch. Rational Mech. Anal., 33 (1969), 246-248.

5. M. Schiffer, $A$ method of variation within the family of simple functions, Proc. London Math. Soc., 44 (1938), 432-449.

6. G. Schober, Univalent Functions; Selected Topics, Springer-Verlag Lecture Notes Volume 478, New York (1975).

7. G. Whyburn, Topological Analysis, Princeton Math. Series Volume 23, Princeton (1958).

Received May 7, 1977.

BRIGHAM Young UNIVERSITY

Provo, UT 84602 


\section{PACIFIC JOURNAL OF MATHEMATICS}

\section{EDITORS}

RICHARD ARENS (Managing Editor)

University of California

Los Angeles, California 90024

C. W. Curtis

University of Oregon

Eugene, OR 97403

C. C. MOORE

University of California

Berkeley, CA 94720

\section{J. DUGUNDJI}

Department of Mathematics University of Southern California Los Angeles, California 90007

R. Finn AND J. Milgram Stanford University Stanford, California 94305

ASSOCIATE EDITORS
E. F. BECKENBACH

B. H. NeUMANN

F. WOLF

K. YoshidA

\section{SUPPORTING INSTITUTIONS}

UNIVERSITY OF BRITISH COLUMBIA UNIVERSITY OF SOUTHERN CALIFORNIA CALIFORNIA INSTITUTE OF TECHNOLOGY STANFORD UNIVERSITY UNIVERSITY OF CALIFORNIA MONTANA STATE UNIVERSITY UNIVERSITY OF TOKYO UNIVERSITY OF NEVADA, RENO UNIVERSITY OF UTAH NEW MEXICO STATE UNIVERSITY OREGON STATE UNIVERSITY UNIVERSITY OF OREGON

WASHINGTON STATE UNIVERSITY UNIVERSITY OF WASHINGTON OSAKA UNIVERSITY 


\section{Pacific Journal of Mathematics}

\section{Vol. 74, No. $1 \quad$ May, 1978}

Gerald Arthur Anderson, Computation of the surgery obstruction groups

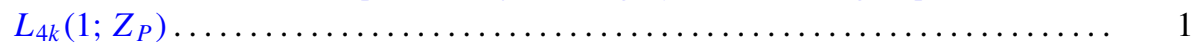

R. K. Beatson, The degree of monotone approximation ................ 5

Sterling K. Berberian, The character space of the algebra of regulated functions . . . 15

Douglas Michael Campbell and Jack Wayne Lamoreaux, Continua in the plane with

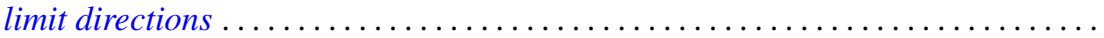

R. J. Duffin, Algorithms for localizing roots of a polynomial and the Pisot

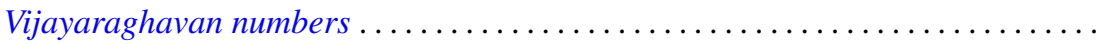

Alessandro Figà-Talamanca and Massimo A. Picardello, Functions that operate on

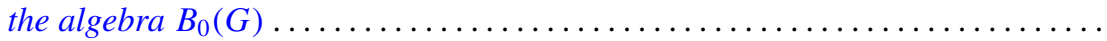

John Erik Fornaess, Biholomorphic mappings between weakly pseudoconvex

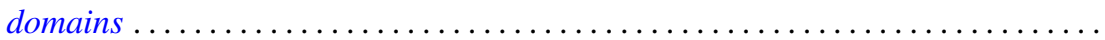

Andrzej Granas, Ronald Bernard Guenther and John Walter Lee, On a theorem of S.

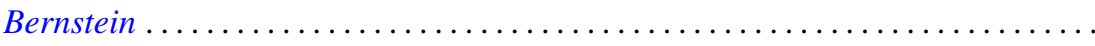

Jerry Grossman, On groups with specified lower central series quotients . .........

William H. Julian, Ray Mines, III and Fred Richman, Algebraic numbers, a constructive development . . . . . . . . . . . . . . . . . . . . . . .

Surjit Singh Khurana, A note on Radon-Nikodým theorem for finitely additive

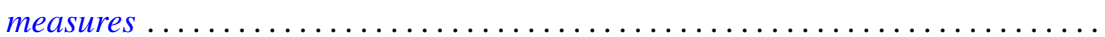

Garo K. Kiremidjian, A Nash-Moser-type implicit function theorem and nonlinear

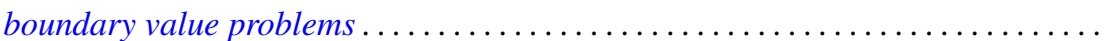

Ronald Jacob Leach, Coefficient estimates for certain multivalent functions ....

John Alan MacBain, Local and global bifurcation from normal eigenvalues. II . . 133

James A. MacDougall and Lowell G. Sweet, Three dimensional homogeneous algebras...

John Rowlay Martin, Fixed point sets of Peano continua ......

R. Daniel Mauldin, The boundedness of the Cantor-Bendixson order of some analytic sets...

Richard C. Metzler, Uniqueness of extensions of positive linear functions ..

Rodney V. Nillsen, Moment sequences obtained from restricted powers . .

Keiji Nishioka, Transcendental constants over the coefficient fields in differential elliptic function fields...

Gabriel Michael Miller Obi, An algebraic closed graph theorem

Richard Cranston Randell, Quotients of complete intersections by $\mathbf{C}^{*}$ actions . . 221

Bruce Reznick, Banach spaces which satisfy linear identities . .

Bennett Setzer, Elliptic curves over complex quadratic fields...

Arne Stray, A scheme for approximating bounded analytic functions on certain subsets of the unit disc.

Nicholas Th. Varopoulos, A remark on functions of bounded mean oscillation and bounded harmonic functions. Addendum to: "BMO functions and the

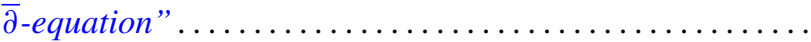

Charles Irvin Vinsonhaler, Torsion free abelian groups quasi-projective over their

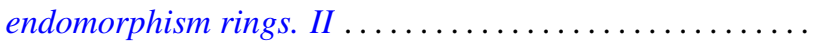

Thomas R. Wolf, Characters of $p^{\prime}$-degree in solvable groups ... 